VALENTINA ROGNOLI

DEPARTAMENTO DE DISEÑO

POLITECNICO DI MILANO

MILÁN, ITALIA

VALENTINA.ROGNOLI@POLIMI.IT

CAMILO AYALA GARCÍA

DEPARTAMENTO DE DISEÑO

UNIVERSIDAD DE LOS ANDES

BOGOTÁ, COLOMBIA.

C.AYALA954@UNIANDES.EDU.CO
Fecha de recepción: 22/01/2018

Fecha de aceptación: 27/03/2018

Cómo citar: Rognoli, V. \& Ayala García, C. (2018).

Materia Emocional. Los materiales en nuestra

relación emocional con los objetos. RChD:

creación y pensamiento, 3(4), 1-15.

DOI: 10.5354/0719-837X.2018.50297

Revista Chilena de Diseño,

RChD: creación y pensamiento

Universidad de Chile

2018, 3(4)

http://rchd.uchile.c

\section{Materia emocional. Los materiales en nuestra relación emocional con los objetos}

\author{
Emotional matter. \\ Materials in our emotional relationship with objects
}

Resumen. Cuando interactuamos con productos, relaciones emocionales se activan. Los diseñadores deben ser conscientes de estas relaciones para crear mejores y significativos productos. Durante las últimas dos décadas, una serie de investigaciones alrededor de las emociones que ocurren entre los productos y sus usuarios han revelado que los materiales juegan un papel importante en el desarrollo de estas relaciones emocionales. El presente artículo evidencia los diferentes antecedentes teóricos alrededor del tema de las emociones en el campo del diseño, focalizándose en la influencia que ejercen los materiales dentro de las relaciones emocionales que se crean entre los productos y quienes los utilizan.

Adicionalmente, presentaremos dos clases de materiales emergentes conocidos como Materiales DIY y Materiales ICS, y la relevancia que estos representan en la disciplina del diseño gracias a la capacidad de convertirse en activadores emocionales, tanto en los usuarios como en quienes los crean. A diferencia de los materiales tradicionales desarrollados por las ciencias y la ingeniería, estos materiales emergentes establecen y mantienen una relación emocional con el diseñador durante sus fases de desarrollo. Estos lazos exaltan las cualidades expresivo-sensoriales de los materiales, por encima de las propiedades físicas y las convierten en características esenciales del proyecto. Palabras clave: emociones, dimensión expresivo-sensorial, materiales, materiales emergentes.

Abstract. When people interact with products, emotional relationships arise. Designers must be aware of those relationships to create meaningful products. In the last two decades, research around the emotions occurring between products and users revealed how materials play a crucial role in the development of emotional relationships. This article presents the theoretical background around the investigations of emotions in the design field, focusing on the influence materials have between the products and the user's emotional relationships. Besides, we will present two classes of emerging materials known as DIY-Materials and ICS-Materials and the relevance they have in the design field, as they are enablers of emotions, not only to the user but also to the designers who create them. Different from the traditional materials produced by science and engineering, those emerging materials maintain a healthy emotional relationship with the designers during the development phase. These ties exalt the expressive-sensorial qualities of the materials over the physical properties and make them essential components of the whole project.

Keywords: emotions, emerging materials, expressive-sensorial dimension, materials. 


\section{Introducción}

Todos los seres humanos sentimos y experimentamos emociones cotidianamente. Muchas de estas emociones se derivan de todo aquello con lo que entramos en contacto de manera voluntaria o casual: cuando leemos un libro, vemos una película, asistimos a una exhibición o compartimos una cena. La lista de actividades que se realizan a diario que pueden desatar o no emociones podría ser infinita. Sin embargo, de aquello que no nos damos cuenta es cuan importantes pueden llegar a ser para nuestras vidas, las emociones provocadas por los objetos que nos rodean y con los cuales interactuamos. La emoción es un aspecto importante en nuestra relación cotidiana con los artefactos, bien sea cuando estos son utilizados o cuando nos disponemos a diseñarlos. La forma de los artefactos y los materiales con los que estos son realizados, estimulan no solo la percepción por parte de los usuarios, sino que reflejan y evidencian la capacidad del diseñador por crear su expresión (Rognoli y Levi, 2011).

Las emociones son elementos fundamentales que componen el esquema de la sensorialidad y la expresividad dado que comparten sus mismos orígenes, es decir, derivan de los sentidos, de los mecanismos sensoriales y son elaborados por el sistema mente-cerebro (Biondi et al., 2009). Barile, 2007 afirma:

El sentir podría ser quizás el aspecto esencial de la emoción; sin el cual solo se podría imitar el comportamiento emocional mas no probarlo en primera persona. Aquello que distingue una emoción con respecto a otros estados mentales, es el arraigamiento en el cuerpo, lo que permite particularmente la experiencia de sentir la emoción misma (p.5).

Aquello que pensamos es provocado por aquello que sentimos. Por ende, es fundamental en el momento de diseñar un proyecto, considerar la estrecha relación que existe entre el pensamiento y el sentimiento.

El presente artículo busca evidenciar la importancia de los mecanismos emocionales en el campo del diseño, específicamente aquellos suscitados por los materiales. A través de una revisión actualizada de la literatura internacional alrededor del tema y manteniendo siempre una perspectiva multi-disciplinar, buscaremos resaltar el rol que cumplen los materiales en esta dinámica. Hablaremos de la dimensión expresivo-sensorial de los materiales y de las cualidades sensoriales que la componen. Estas cualidades o características son la base del mecanismo emocional de la materia, son elementos fundamentales que el diseñador debe conocer a fondo para poder diseñar y proyectar. Solo así podrá activar y resaltar emociones en los diferentes usuarios mediante la apropiación del concepto de "Materials Experience" o experiencia material (Karana et al., 2014; Karana et al., 2015).

Adicionalmente presentaremos dos tipos de materiales emergentes conocidos a nivel internacional como Materiales DIY (Materiales-auto-producidos) por un lado y los Materiales Ics (Materiales Interactivos, Conectados e Inteligentes) por el otro. Ambos grupos de materiales son relevantes desde el punto de vista emocional, ya que, a diferencia de los materiales tradicionales provenientes de la ciencia y la ingeniería, estos son desarrollados por diseñadores en asociación con otras disciplinas. Esta particularidad hace que, desde la concepción misma del material, se tengan en cuenta sus relaciones con los usuarios, se exalten sus cualidades expresivo-sensoriales, 
por encima de sus propiedades físicas y se conviertan en parte integral del proyecto de diseño. Explicaremos como estos materiales se centran en la experiencia que tienen las personas que los crean durante su proceso de desarrollo, al igual que en la experiencia que se crea en aquellos que los ven y utilizan embebidos en diferentes productos. De este modo ilustraremos cómo los diseñadores pueden utilizar los materiales para la realización de sus proyectos, valorizando el aspecto emocional.

\section{Las emociones en el diseño}

Durante las dos primeras décadas del siglo XXI diferentes investigadores y académicos de la disciplina del diseño han iniciado a estudiar las emociones. Estos estudios han proporcionado teorías, herramientas y métodos que han permitido a los diseñadores definir y tratar de cuantificar las emociones que evocan los productos.

La relación emocional entre los usuarios y los objetos se ha convertido en un extenso campo de investigación en áreas como el diseño emocional, el diseño de experiencias a través del producto y las experiencias de uso. En estas áreas, la investigación ha alcanzado una escala global (Desmet et al., 2001; Demirbilek y Sener, 2003; Norman, 2004; Desmet y Hekkert, 2007; Schifferstein y Hekkert, 2008; Chitturi, 2009). Los diferentes investigadores en estas áreas se han dedicado particularmente a clasificar y esquematizar los sentimientos y las emociones (Desmet, 2003); en entender los conceptos emocionales en los usuarios que interactúan con los productos (Demir, 2008; Norman 2004); la valoración de los diferentes patrones emocionales presentes en las interacciones entre seres humanos y productos (Demir et al., 2009) y el desarrollo de herramientas para medir la acción emocional en el campo del diseño (Desmet, 2003).

Hoy sabemos gracias a estos estudios, que cada decisión de diseño puede crear un efecto emocional. El entendimiento de las emociones en los usuarios como evidenciamos anteriormente, puede ayudar al diseñador a anticipar el impacto emocional y del mismo modo evitar aquellas emociones no deseadas, como pueden serlo, por ejemplo, los sentimientos de desagrado como respuesta frente a un nuevo producto diseñado (Desmet y Hekkert, 2009). Es importante para un diseñador crear productos capaces de evocar experiencias que el usuario de algún modo espera experimentar.

En los últimos años, algunas investigaciones se han concentrado en el papel que cumplen las emociones en el comportamiento sostenible. Chapman $(2005 ; 2014)$ ha evidenciado como las emociones provocadas por los objetos está determinada por la durabilidad misma de estos. En realidad, el diseñador debe garantizar la sostenibilidad de los objetos creando y proyectando su significado junto a su aspecto emocional. Según su propia visión, el diseñador debe poder transformar la relación entre los usuarios y los objetos para que se cree una especie de matrimonio entre ambos; una relación de crecimiento mutuo duradero contrario a una simple historia pasajera como sucede actualmente con los productos de consumo masivo. Ortiz por su parte (2017) recientemente ha estudiado cómo las emociones placenteras suscitadas por los objetos son elementos fundamentales en la interacción que se produce entre el usuario y el objeto. Estas emociones deben ser consideradas por el diseñador si aspira proyectar de manera sostenible. Ortiz ha caracterizado experiencias placenteras sugiriendo estrategias para diseñar productos que las puedan provocar. Según el autor, el uso de estas 
Figura 1. Tazones de diferentes materiales utilizados para estudiar la relación entre reacción emocional y el material por diferentes usuarios (Crippa et al., 2012).

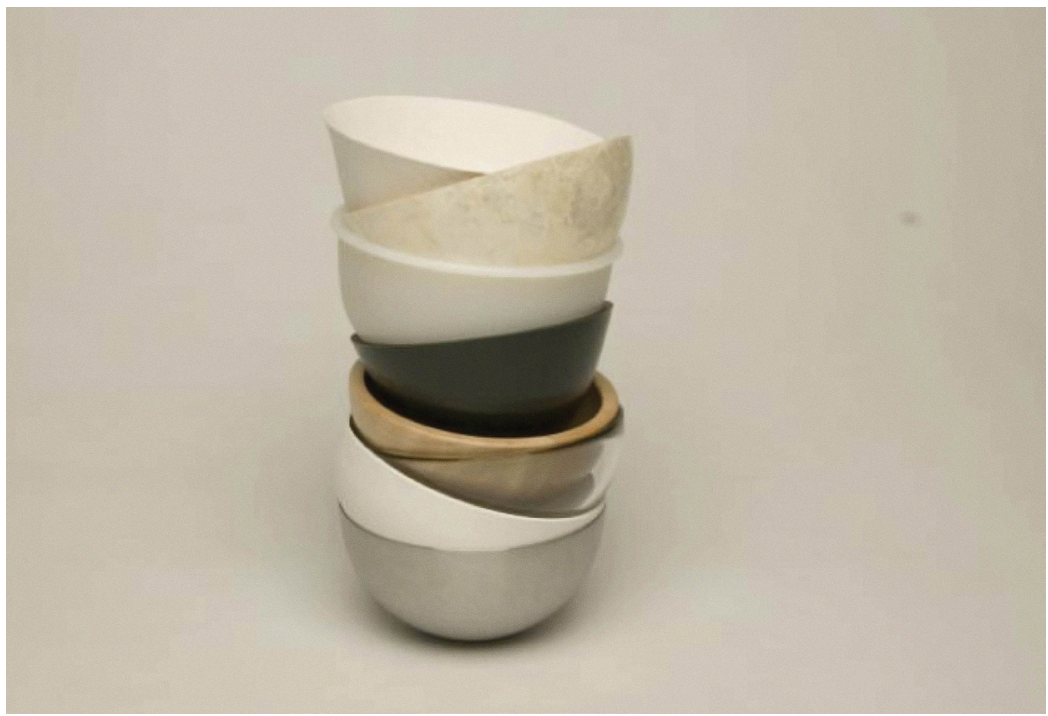

estrategias puede seguramente permitir al diseñador, generar productos que, conectándose emocionalmente con los usuarios, pasen de ser simples objetos para convertirse en elementos de conexión e interacción entre las personas y el medioambiente. El apego emocional tiene unas implicaciones importantes en términos de sostenibilidad, ya que los productos que gozan de estos atributos tienden a tener ciclos de vida mucho más largos.

El papel que juegan los materiales en las dinámicas emocionales entre los usuarios y los objetos es ya objeto de estudio. La literatura en el campo del diseño evidencia que las formas, los colores y los materiales, son elementos fundamentales de los productos y los toma como elementos estratégicos para evocar emociones (Ürgen, 2006; Chang y Wu, 2007; Kim y Boradkar, 2002). Se ha dicho igualmente que el impacto emocional de un producto depende tanto de sus cualidades materiales, sus propósitos, sus significados y sus expresiones, como lo que hace y deja de hacer (Desmet, 2010). Algunos investigadores se han concentrado en estudiar como los materiales puedan influenciar la experiencia de uso del producto y como puede ser posible describir emotivamente algunas cualidades del mismo que permiten determinar los estados de ánimo de los usuarios (Van Kesteren, 2008; Crippa et al., 2012) (Figuras 1 y 2). Otras investigaciones se han llevado a cabo focalizándose principalmente en la sorpresa como emoción importante, muchas veces determinada por el tipo de material utilizado para dar forma al producto (Ludden et al., 2008; Ludden et al., 2012). Encontramos por último, investigaciones recientes que estudian la posibilidad que tienen los materiales para crear relaciones duraderas con los objetos, que como decíamos en la sección anterior, tienden a ser coherentes con las actuales teorías relativas a la sostenibilidad (Rognoli y Karana, 2014; Karana et al., 2017).

\section{Las emociones a través de los materiales}

Si bien en el capítulo anterior nos hemos enfocado en el deber del diseñador por entender las emociones y las capacidades que estas tienen para el desarrollo de productos con mayor significado, ahora nos concentramos en explicar un poco el rol que los materiales ejercen en este complejo mundo de interacciones emocionales entre el usuario y el producto. En los últimos 


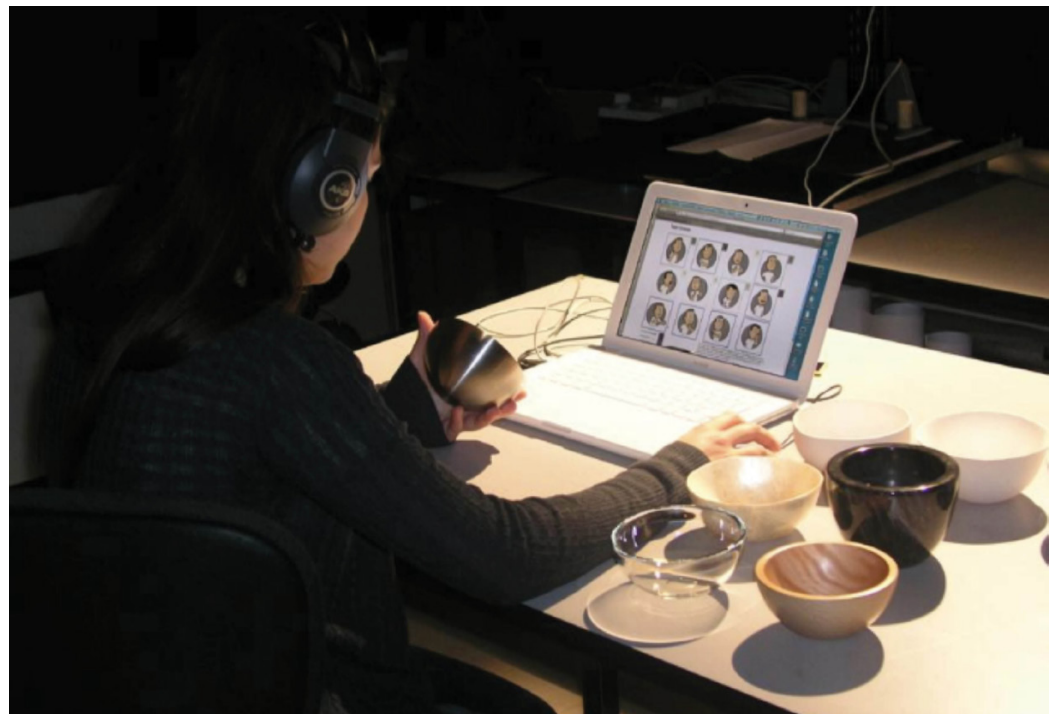

años, los materiales han sido objeto de estudio por parte de los diseñadores ya que no solo son tenidos en cuenta por sus características funcionales, sino por la capacidad que tienen para inspirar y generar emociones. Cada vez más y con mayor frecuencia, vemos a diseñadores que emplean las diferentes cualidades de los materiales para crear diferentes experiencias expresivas y sensoriales en conjunto con nuevas formas y lenguajes de producto. Como asegura Jordan (2002), los materiales mediante los cuales se realiza un producto, pueden ejercer un papel determinante en cuan agradable o desagradable puede ser para aquellos que interactúen con él.

Los materiales han adquirido un rol que no consiste solamente en proveer propiedades físicas o de ingeniería. Este nuevo rol es de cierta manera invisible, pues ya no usamos los objetos con fines puramente funcionales; nos interesa más un nivel emocional y los materiales contribuyen en conseguirlo (Lefteri, 2007).

Los materiales se involucran en los diferentes procesos de interacción con las personas y son capaces de influenciar la relación que se crea entre los usuarios y los productos (Ashby y Johnson, 2002; Van Kesteren et al., 2005). Norman, por su parte (2004) afirma que los materiales de los productos influencian el nivel visceral de las emociones y son capaces de despertar inmediatamente reacciones de efecto sorpresa. En efecto, los diseñadores creen que los materiales y los procesos son aquel espacio donde se puede agregar espíritu al diseño y a la forma; el lenguaje visual y la emoción del producto estará siempre ahí, por encima de su viabilidad productiva o sus costos de fabricación (Pedgley, 2009). Los diseñadores deben seleccionar materiales (o auto-producirlos) para la realización de sus productos considerando la capacidad emocional de estos. De esta manera lograrían diseñar productos portadores de un valor emocional superior. Los diseñadores tienen la oportunidad de influenciar la relación emocional que se crea entre los usuarios y los productos a través del uso adecuado de los materiales. Por esta razón varios investigadores han declarado un interés en examinar a fondo las respuestas emocionales de las personas, focalizándose en aquellas provocadas por los materiales. El
Figura 2. Participante evaluando nueve tazones de diferentes materiales (Crippa et al., 2012). La evaluación se realizó utilizando la herramienta PrEmo. Premo Tool, es una herramienta diseñada por Peter Desmet para medir las emociones evocadas por los productos (www.premotool.com). 
resultado de estas investigaciones ha servido para definir diferentes estrategias que ayudan a desarrollar productos capaces de suscitar emociones como la curiosidad, el amor, la felicidad, etc. (Karana, 2009).

Materiales, tecnologías y acabados son hoy en día considerados estímulos y fuentes de inspiración para los diseñadores que son capaces de crear nuevas experiencias sensoriales junto a nuevos lenguajes formales. El fenómeno consolidado internacionalmente de las Materiotecas como fuente de inspiración e información acerca de los materiales para el diseño, ha contribuido notablemente a focalizar la atención en los materiales como verdaderos motores de innovación, generadores de experiencias y exaltadores de emociones (Rognoli, 2005). Esto a su vez ha abierto las puertas para que la investigación en diseño haya comenzado a concentrar sus esfuerzos en entender el fenómeno y desarrollar diferentes teorías alrededor de los aspectos de los materiales en la disciplina del diseño. Las áreas principales de investigación en este campo son: La caracterización expresivo-sensorial de los materiales (Rognoli, 2004; Rognoli y Levi, 2004; Rognoli 2010; Karana et al., 2009). El significado de los materiales (Karana, 2009; Karana, Hekkert, 2010), La selección de los materiales (Ashby y Johnson, 2002; Pedgley, 2009; Van Kesteren, 2008), Educación en materiales para el diseño (Pedgley, 2016; Rognoli, 2010; Ayala-García, 2014, 2015) y finalmente La experiencia material (Karana et al., 2015).

Diferentes contribuciones de estos autores han construido las bases para sustentar lo que hoy conocemos como La Experiencia Material. Esta fue definida por Karana et al. (2008) como la experiencia que tienen las personas con y a través de los materiales de un producto. En su descripción original la experiencia material consta de tres componentes experienciales: la experiencia estética o sensorial (cuando percibimos el material frío o caliente, suave o rugoso, brillante u opaco, etc.), la experiencia de significado (cuando pensamos que el material es moderno o clásico, cómodo o incomodo, elegante o común, etc.) y la experiencia emocional (cuando el material nos hace sentir asombrados, sorprendidos, felices, aburridos o tristes, etc.). Más adelante Giaccardi y Karana (2015) ampliaron la definición de experiencia material adicionando un cuarto componente a nivel de actuación. Según las autoras, la definición de experiencia material debe reconocer el rol activo que tienen los materiales no solo en la forma como se vinculan interiormente con nosotros y los objetos, sino en el rol que tienen estableciendo relaciones con el hacer y las formas como se elaboran las cosas.

Diseñar con un material se convierte pues en una experiencia de entendimiento para conocer y comprender sus cualidades únicas y limitaciones con respecto a otros materiales. Este entendimiento comienza desde el primer encuentro entre el diseñador y el material, y se extiende hasta la materialización del producto al final del proceso. Es una especie de proceso exploratorio de creación y evaluación constante que se logra a través de pensar con el material donde la manipulación de la materia a través de una interacción divertida, ingenua y creativa, puede llevar a desarrollar innovación y originalidad en el diseño (Parisi, Rognoli y Sonneveld, 2017).

\section{Emociones y materiales emergentes}

Como parte de esa exploración reciente en la que diseñadores y creativos se han visto involucrados para generar nuevos lenguajes de producto con la ayuda de los materiales, nos encontramos con dos fenómenos que están siendo objeto de estudio; los Materiales DIY (Rognoli et al., 2015; Ayala-García 
et al., 2017) y los Materiales Ics (Rognoli et al., 2016; Parisi et al., 2018). Estos materiales comportan y representan una dimensión emotiva relevante y son objeto de estudio dentro de la experiencia material. Principalmente, porque se centran en la experiencia que tienen las personas que los crean durante su proceso de desarrollo, al igual que en la experiencia que se crea en aquellos que los ven y utilizan embebidos en diferentes productos.

\section{Materiales DIY}

En la década anterior, una aproximación hacia los materiales por parte de los diseñadores emergió trayendo consigo una nueva dimensión en cuanto a la relación entre los diseñadores, la tecnología, los procesos productivos y los materiales. Esta novedosa aproximación evoca las practicas conocidas como "Hágalo usted mismo", muy populares en los años setenta, que definieron una contracultura respecto a los crecientes procesos de industrialización (Anderson, 2012), donde la combinación del hacer manualmente, la artesanía y la fabricación de pequeñas series (Gershenfeld, 2012; Kuznetsov y Paulos 2010; Tanenbaum et al., 2013) se reconocen como el renacimiento de la artesanía. (Bardzell et al., 2012; Bean y Rosner, 2012; Bettiol y Micelli, 2014; Sennett, 2008). El movimiento DIY (Do-It-Yourself) o 'Hágalo Usted Mismo', se ha expandido más allá de la creación de productos y se ha extendido a los materiales con los cuales estos productos son realizados (Brownell, 2015). En una publicación reciente, Rognoli junto a otros académicos, introdujeron el concepto de Materiales DIY definiéndolos como "aquellos materiales creados a partir de prácticas colectivas o individuales, a menudo desarrollados con técnicas y procesos inventados por el diseñador. Estos pueden ser materiales completamente nuevos, modificados o incluso versiones modificadas de materiales existentes" (Rognoli et al., 2015; Rognoli y Ayala-García, 2018). La aproximación a los Materiales DIY es cada vez más usada por los diseñadores por diferentes razones en las que se incluyen elementos como: el interés por desarrollar futuros sostenibles, el deseo por obtener lenguajes únicos de expresión material o reaccionar de manera activa contra la producción en masa. Estas prácticas de auto-producción de materiales, promueven el desarrollo del conocimiento a través de la acción (Schön, 1983). Por ende, el conocimiento que se desarrolla dentro de la mente del diseñador, genera en él diferentes emociones. Estas emociones obtenidas a través de la experiencia directa con el material, si son interpretadas de manera consciente por el diseñador, podrán ser transmitidas a sus creaciones. Este proceso de realización de materiales a través de la manipulación de ingredientes y elementos de composición es conocido como el 'pensar con la mano' (Nimkulrat, 2012). Es un elemento de desarrollo intelectual bastante interesante (Sennett, 2008, pp. 149-153), pues se convierte en un proceso dinámico de aprendizaje y entendimiento a través de la experiencia material (Gray y Burnett, 2009, p. 51) y por ende se convierte en la herramienta principal para diseñar con materiales. El involucramiento personal y físico en la creación de objetos, crea un lazo profundo entre el objeto y quien lo idea (Salvia, 2016). Si algo ha sido realizado con las propias manos, se trata con cuidado, tiene más valor frente a nuestros ojos y nos gusta más. Estamos dispuestos a aceptar imperfecciones y estéticas alternativas. Esto no sucede cuando se trata de producciones industriales, donde estamos acostumbrados a la perfección, sin defecto alguno y producido de inmediato. La relación emocional que se crea entre algo que ya ha sido fabricado (posiblemente por una máquina y en serie) o aquella 
que se gesta cuando se realiza algo con las propias manos, son completamente distintas. Por consiguiente, podemos decir que los materiales autoproducidos habilitan al usuario/diseñador a desarrollar nuevas relaciones emocionales con los materiales. Estas se transmiten a través de las diferentes dimensiones estéticas de dichos materiales (Rognoli et al., 2016; Ayala-García y Rognoli, 2017). El importante crecimiento de este fenómeno de Materiales DIY ha requerido la concentración y profundización de estudios, que ayuden a comprender y analizar dicho fenómeno posteriormente. Por esta razón, hemos iniciado un recorrido de investigación doctoral alrededor de este fenómeno, su aproximación por parte de los diseñadores y su sistema de clasificación (Ayala-García, en desarrollo). Según Ashby y Johnson (2002) "la clasificación es el primer paso en la organización de cualquier esfuerzo científico. Los fundadores de la biología, la zoología y la geología fueron quienes crearon los sistemas de clasificación". Inspirados en una de las primeras clasificaciones de la biología del siglo XVII, realizada por el célebre botánico, zoólogo y físico Carolus Linneaus denominado Systema Naturae (Linnaeus, 1758), se generaron para los Materiales DIY cinco categorías de clasificación llamados reinos (Figura 3). Estos reinos se basan principalmente en los principales elementos que los componen y su origen (Ayala-García et al., 2017). Estos reinos son:

Reino Vegetabile. Cuando los elementos principales derivan de plantas y hongos, estos materiales son clasificados dentro del reino vegetal. Los materiales al interior de este reino se diferencian de los demás principalmente porque estos son realizados mediante el cultivo o el crecimiento. Los diseñadores que crean materiales pertenecientes a esta categoría frecuentemente colaboran con biólogos o agricultores.

Reino Animale. se refiere a todos los elementos derivados de animales y bacterias. Estos materiales pueden ser desarrollados bien sea colaborando con diferentes organismos (Ej. co-creando con abejas para producir un material) o utilizando partes de este como su pelo o sus huesos.

Reino Lapideum. Este contiene todos aquellos materiales auto producidos cuyos elementos principales son minerales: piedras, arena, cerámica, arcilla, etc. Muchos casos existentes combinan elementos provenientes de otros reinos como la lana o las fibras de algodón, aunque en un porcentaje relativamente menor comparado con el constituyente principal. Otra característica de este reino es la fuerte conexión con la artesanía, probablemente gracias a que este tipo de elementos ha tenido siempre una larga tradición en nuestra cultura material. Reino Recuperavit. Incluye todos los elementos que son considerados como desperdicio, que sin embargo pueden ser transformados en una valiosa fuente de recursos. A menudo provienen de los plásticos, los metales o desechos orgánicos, que en algunas ocasiones provienen de desechos de la industria y la producción en masa. Es hasta el momento el reino con mayor número de casos de estudio encontrados.

Reino Mutantis. Incluye todos los materiales auto producidos creados a través de la hibridación de fuentes industriales, interactivas (mediante el soporte de electrónica de código abierto) o fuentes inteligentes (que pueden cambiar sus propiedades físicas, pueden intercambiar energía o intercambiar materia). Se incluyen también mezclas de diferentes elementos de otros reinos que evolucionan hacia un material específico con la ayuda de una tecnología particular. Esta hibridación representa un cambio significativo de paradigma comparado con los demás reinos de los materiales auto producidos. 


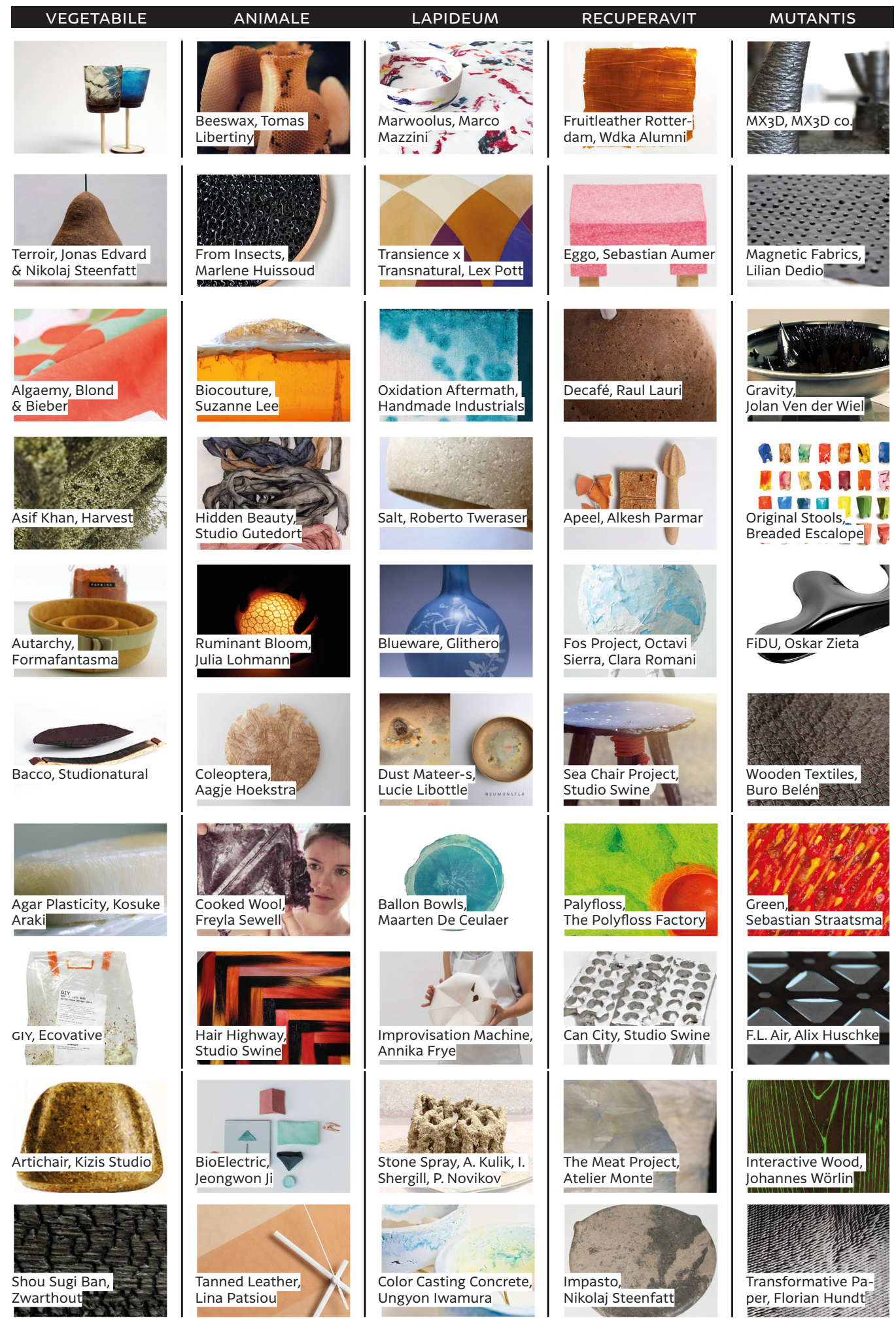

Figura 3. Selección de casos clasificados dentro de los cinco reinos de los Materiales DIY (Ayala García et al., 2017). 
Figura 4. Proceso de experimentación y elaboración de un Material DIY (Pánico, 2017).
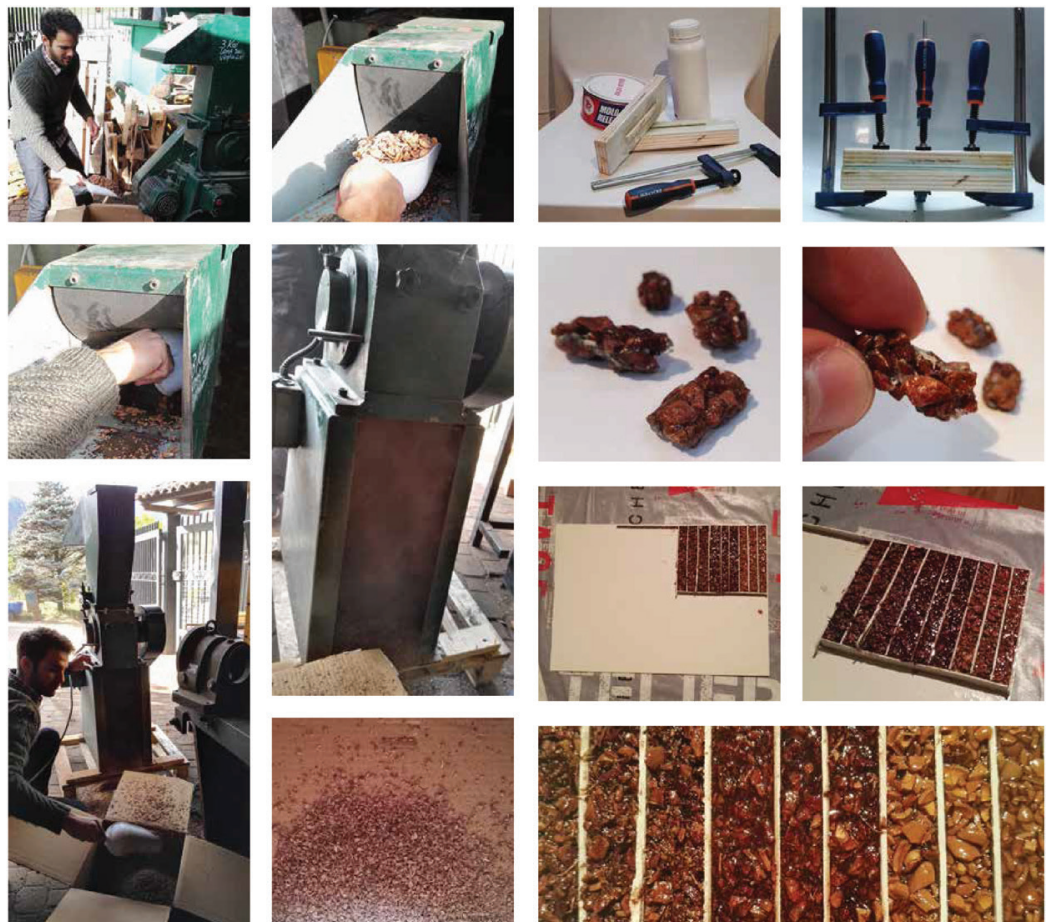

Los primeros estudios realizados alrededor de este sistema de categorización de los Materiales DIY han demostrado dos elementos fundamentales: primero, surge evidencia que, en los diferentes casos de estudio clasificados en cada reino, existe un valor emocional relacionado al tipo de auto producción y experimentación. Los casos de estudio clasificados al interior del reino Recuperavit, por ejemplo, tienen los mismos motores emocionales que empujan el desarrollo de los materiales. Segundo, hemos encontrado que quienes se proponen a experimentar para crear Materiales DIY, al conocer su clasificación, direccionan sus procesos de creación mediante el descubrimiento y exploración de elementos y recursos impensables y originales con respecto a las clasificaciones tradicionales de los materiales. Este descubrimiento genera en el diseñador una serie de emociones de gran valor, crea una relación íntima con el material y mediante el diálogo que surge entre ambos, nacen proyectos de un alto nivel de originalidad con un gran potencial de innovación en las fases sucesivas de desarrollo (Figuras 4 y 5 ).

\section{Materiales ICS}

Al igual que en el caso de los Materiales DIY, los materiales inteligentes, también han conseguido nuevas categorizaciones influenciados por la computación, el internet de las cosas y la hibridación entre el diseño y la ciencia de la comunicación. Estos nuevos materiales están sobrepasando aquellos tradicionales ya que presentan grados adicionales de inteligencia. Ics es el acrónimo para describir los materiales interactivos, conectados e inteligentes (Interactive, Connected and Smart, por sus siglas en inglés) (Rognoli, 2017; Parisi et al., 2018). Si bien recientes investigaciones se centran principalmente en la experimentación de estos materiales, existe una necesidad de reflexionar en su definición, clasificación y sobre todo en entender los diferentes niveles de experiencia con el usuario y las posibles emociones que se puedan desatar de estas interacciones. 

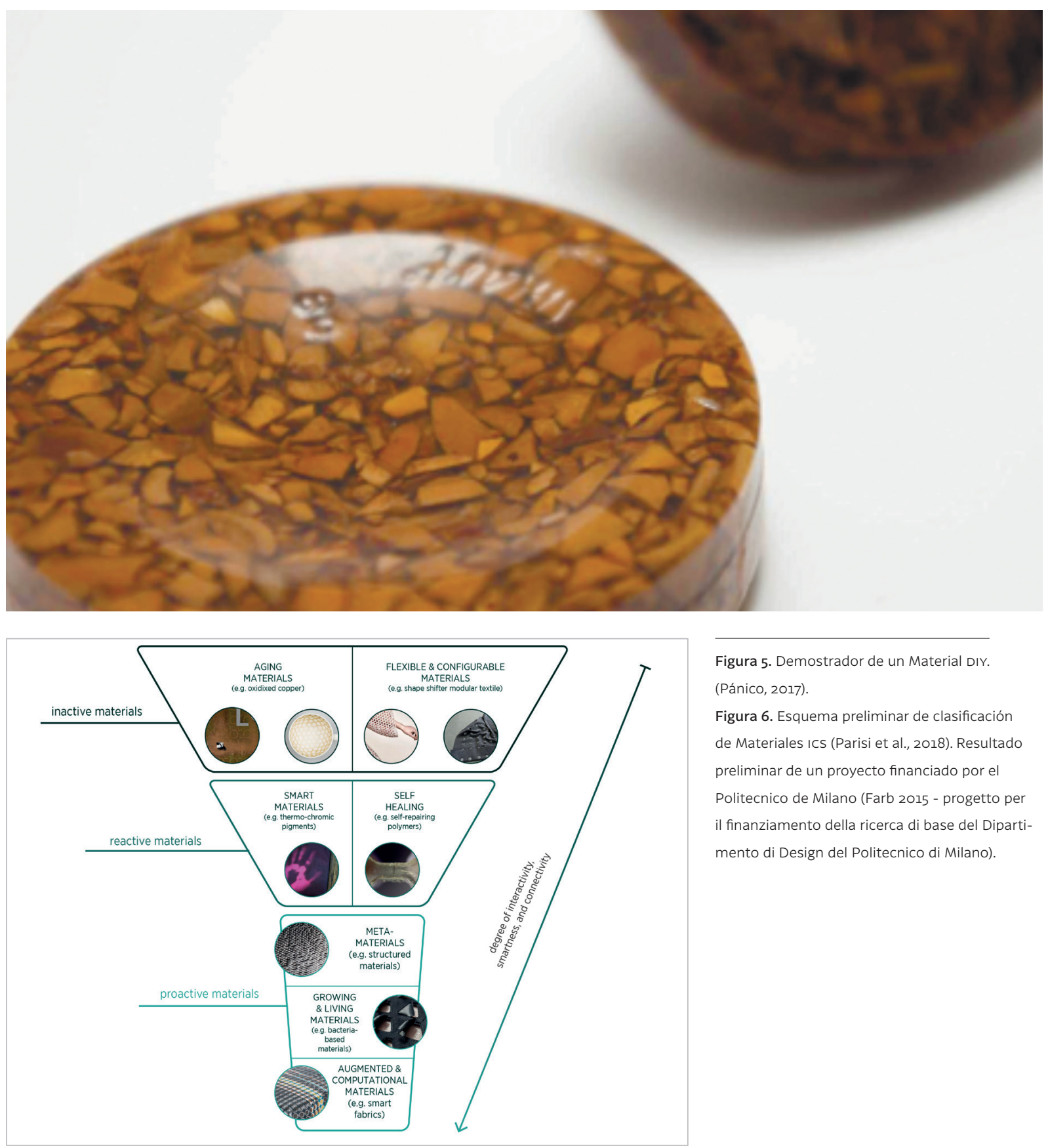

Figura 5. Demostrador de un Material DIY. (Pánico, 2017).

Figura 6. Esquema preliminar de clasificación de Materiales ICS (Parisi et al., 2018). Resultado preliminar de un proyecto financiado por el Politecnico de Milano (Farb 2015 - progetto per il finanziamento della ricerca di base del Dipartimento di Design del Politecnico di Milano).

Una primera aproximación en la clasificación de estos materiales, resultado de un trabajo académico (Figura 6) ha logrado evidenciar posibles categorías. Estas categorías, elaboradas estrechamente bajo las nociones de experiencia material descritas anteriormente, permiten comprender mejor su naturaleza, sus grados de inteligencia y sobre todo, su nivel de interacción con otras entidades que pueden ser humanas o no humanas. Estas interacciones, aunque sean en algunos casos entre objetos o entre materiales, derivarían eventualmente en una serie de emociones por parte de quienes interactúen con estos o los objetos con estos producidos. Estas emociones 
serán seguramente la base de estudios consecutivos dado que muchas de las relaciones que tenemos actualmente con la tecnología y lo que esta nos hace sentir o percibir, no es claro aún por las diferentes ramas de la ciencia que las estudian. Las categorías preliminares son:

Materiales Inactivos. Son aquellos materiales tradicionales donde no existe una interacción explícita o que permiten un bajo nivel de interacción que no demuestra la habilidad de reaccionar o conectarse; en otras palabras, se comportan de manera pasiva.

Materiales Reactivos. se incluyen materiales inteligentes o combinaciones de materiales inactivos con componentes inteligentes como, por ejemplo, los pigmentos termo-cromáticos. Estos evidencian transformaciones de propiedades mediante cambios de color o forma, producto de un estímulo externo. Materiales Proactivos. están representados por sistemas complejos e inteligentes basados en combinaciones de materiales inactivos o reactivos junto con tecnologías digitales, computacionales o electrónicas. Estas tecnologías se incorporan en forma de sensores o activadores conectados con algún tipo de computador externo o interno. Las oportunidades que ofrecen las tecnologías inteligentes, ampliando y complejizando las diferentes funciones, deben ser consideradas por los diseñadores por el potencial que tienen para definir nuevos significados y lenguajes estéticos. La hibridación entre el diseño y la inteligencia artificial, al igual que la fusión entre materiales tradicionales con tecnologías de computación, abren nuevas perspectivas en la disciplina del diseño. Estas nuevas fronteras invitan a diseñadores e investigadores a explorar nuevas expresiones materiales cargadas de experiencias estéticas, sensoriales y de interacción. A través del estudio de los Materiales ICS se pueden conocer y comprender las diferentes herramientas y tecnologías actualmente disponibles para tales fines. El entender como esta dimensión moderna del diseño se sobrepone a las dimensiones más tradicionales ayudará seguramente a desarrollar productos y servicios más interactivos, conectados e inteligentes, con el apoyo de la materia. Esta cualidad interactiva avanzada que proponen este tipo de materiales, podría seguramente transformar las lógicas actuales de experimentación que se hacen en el mundo de la computación, llevando a la maduración de ciertas tecnologías y alcanzando niveles de creación de producto inimaginables hasta el momento.

\section{Conclusiones}

En este artículo hemos realizado un recorrido que se basa en nuestra experiencia como investigadores en una de las ramas relativas al diseño de las emociones. Esta rama que se ocupa particularmente de estudiar y entender el rol que los materiales ejercen en estos mecanismos emocionales, creemos que tiene un gran potencial de desarrollo futuro. El recorrido parte de la definición de emoción, cuyo significado abarca un complejo sistema de aportes provenientes de diferentes disciplinas. Creemos que para el diseñador es importante conocer y dominar estas definiciones, si se quiere diseñar teniendo en consideración las emociones y las reacciones emotivas de los usuarios. Todos los materiales articulan emociones y tienen un rol preciso en el proyecto que va más allá de dar forma concreta al objeto. Los materiales tienen la capacidad de caracterizarlo con cualidades que son propias del estos y pueden contribuir notablemente en la percepción final del producto y a las relaciones emocionales que se generen durante su ciclo de vida. Estamos convencidos que la parte más importante de la experiencia material que un usuario desarrolla al 
interactuar con un objeto, es la dimensión expresiva-sensorial, ya que es a través de los sentidos que comienza la percepción del objeto. Esta percepción se puede transformar en emoción y en atribución de significado. Somos conscientes que estos mecanismos emocionales complejos no deben ser vistos superficialmente o de modo generalizado. Sin embargo, sabemos que el diseñador debe tener claros los puntos de referencia para dar inicio a sus procesos de creación y las cualidades expresivo-sensoriales de los materiales son un punto de referencia fundamental. Concluimos hablando de materiales emergentes y presentando los Materiales DIY y los Materiales ICS; dos aproximaciones muy actuales en el mundo de los materiales. Una de las características comunes que comparten estos materiales emergentes es seguramente la dimensión de experimentación, dado que el diseñador se involucra personal y físicamente con el proyecto. Los materiales no son solamente elementos a seleccionar en una librería física o virtual para dar viabilidad a un proyecto, sino que pueden ser elementos indivisibles del proyecto mismo, garantizando que la experiencia emocional que suscite el producto en las personas, esté supeditada a la transformación de la materia y a las nuevas interacciones que esta consiente.

\section{Referencias}

Anderson, C. (2012). Makers-The New Industrial Revolution. Random House Business Books.

Ashby, M., \& Johnson, K. (2002). Materials and Design. The Art and Science of Material Selection in Product Design. Elsevier, UK: Butterworth-Heinemann.

Ayala-Garcia, C. (2014). Experimenting with Materials. - A source for designers to give meaning to new applications. In Proceedings of the Design and Emotion Conference 2014 (pp. 408-417). Bogotá: Universidad de los Andes.

Ayala-Garcia, C. (2015). The Basis of Processes - Experimenting with Food to Re-Shape the Industry Language. In Proceedings of Cumulus Conference, The Virtuous Circle: Design Culture and Experimentation. Milan: McGraw-Hill Education.

Ayala-Garcia, C., \& Rognoli, V. (2017). The new materials aesthetics. DIY-Materials as triggers of new sensorial experiences. The Design Journal, 20, Issue supı: Design for Next: Proceedings of the 12th European Academy of Design Conference, Sapienza. University of Rome, Italy, S375-S389.

Ayala-García, C., Rognoli V., \& Karana E. (2017). Five Kingdoms of DIY Materials for Design. In Proceedings of EKSIG 17 - Alive. Active. Adaptive - Experiential Knowledge and Emerging Materials, 19-20 Jun. The Netherlands.

Barile, E. (2007). Dare corpo alla mente. Milano: Bruno Mondadori.

Bardzell, S., Rosner, D. K., \& Bardzell, J. (2012). Crafting quality in design: integrity, creativity, and public sensibility. In Proceedings of the Designing Interactive Systems Conference (DIS '12) (pp. 11-20). New York, NY: ACM.
Bean, J., \& Rosner, D. K. (2012). Old hat: craft versus design? Interaction, 19(1). New York, NY: ACM, 86-88.

Bettiol, M., \& Micelli, S. (2014). The hidden side of design: the relevance of artisanship. Design Issues, 30(1), 7-18.

Biondi, E., Rognoli, V., \& Levi, M. (2009). Le neuroscienze per il design: la dimensione emotiva del progetto. Milano: Franco Angeli.

Brownell, B. (2015). DIY Design Makers Are Taking On Materials. The Journal of the American Institute of Architects. September 17, 2015 [online] http://www. architectmagazine.com/technology/diy-design-makers-are-taking-on-materials_o - March 2017).

Chang, W., Wu, T. (2007). Exploring Types and Characteristics of Product Forms. International Journal of Design, $1(1), 3-14$.

Chapman, J. (2005). Emotionally Durable Design. Objects, Experiences o Empathy. London: Earthscan.

Chapman, J. (2014). Meaningful Stuff: Toward Longer Lasting Products. In Karana, E., Pedgley, O., \& Rognoli, V. (Eds.) (2014), Materials Experience: Fundamentals of Materials and Design (pp. 135-143). Elsevier, UK: Butterworth-Heinemann.

Chitturi, R. (2009). Emotions by Design: A Consumer Perspective. International Journal of Design, 3(2), 7-17.

Crippa, G., Rognoli, V., \& Levi, M. (2012). Materials and Emotions. A Study on the relations between Materials and Emotions. Industrial Products. In Brassett, J., McDonnell, J., \& Malpass, M. (Eds.). In Proceedings of 8th International Design and Emotion Conference. Central Saint Martin's College of Art \& Design, London, UK. 
Demir, E. (2008). The Field of Design and Emotion: concepts, arguments, tools and current issues. Journal of the Faculty of Architecture METU, 25(1), 135 -152.

Demir, E., Desmet, P. M. A., \& Hekkert, P. (2009). Appraisal Patterns of Emotions in User.

Product Interaction. International Journal of Design, 3(2), 41-51.

Demirbilek, O., \& Sener, B. (2003). Product design, semantics and emotional response. Ergonomics, 46(13/14), 1346-1360.

Desmet, P. M. A., Overbeeke, C. J., \& Tax, S. J. E. T. (2001). Designing products with added emotional value: development and application of an approach for research through design. The Design Journal, 4(1), 32-47.

Desmet, P. M. A. (2003). Measuring Emotions. Development and application of an instrument to measure emotional response to products. In Blythe, M. A., Monk, A.F., Overbeeke, K. (Eds.). Funology, from Usability to Enjoyment (pp. 111-123). Dordrecht: Kluwer Academic Publisher.

Desmet, P. M. A., \& Hekkert, P. (2007). Framework of Product Experience. International Journal of Design, 1 , Issue (1), 57-66.

Desmet, P. M. A., \& Hekkert, P. (2009). Special Issue Editorial: Design \& Emotion. International Journal of Design, 3(2), 1-6.

Desmet, P. M. A. (2010). Three Levels of Product Emotion. In Bouchard, C., Aussat, A., Levy, P., \& Yamanaka, T. (Eds.). KEER: The Proceedings of the Kansei Engineering and Emotion Research International Conference (pp. 238-248), 2-4 March, Paris, France.

Gershenfeld, N. (2012). How to make almost anything. the digital fabrication revolution. Foreign Affairs, 6(91), 43-57.

Gray, C., \& Burnett, G. (2009). Making sense: An exploration of ways of knowing generated through practice and reflection in craft. In Proceedings of the Crafticulation and Education Conference (pp. 44-51). Helsinki, Finland: NordFo.

Jordan, P. (2002). Designing Pleasurable Products. An Introduction to the New Human Factors. London: Taylor and Francis.

Karana, E., Giaccardi E., \& Rognoli, V. (2017). Materially Yours. In Chapman, J. (Ed.), Routledge Handbook of Sustainable Product Design (pp. 269-288). Abingdon: Routledge.

Karana, E., Pedgley, O., \& Rognoli, V. (2015). On Materials Experience. Design Issues, Summer (31:3), 16-27. DOI:10.1162/DESI_a_00335

Karana, E., Pedgley, O., \& Rognoli, V. (Eds.) (2014). Materials Experience: Fundamentals of Materials and Design. Elsevier, UK: Butterworth-Heinemann. (http://store.elsevier.com/Materials-Experience/isbn$9780080993768 /$ )
Karana, E., Hekkert, P., \& Kandachar, P. (2009). Assessing Material Properties on Sensorial Scales. In ASME 2009, Proceedings of the International Design Engineering Technical Conferences and Computers and Information in Engineering. San Diego, USA.

Karana, E., Hekkert, P., \& Kandachar, P. (2008). Materials experience: descriptive categories in material appraisals. In Proceedings of the International Conference on Tools and Methods in Competitive Engineering 2008. Izmir, Turkey.

Kim, D., \& Boradkar, P. (2002). Sensibility Design.

http://new.idsa.org/webmodules/articles/articlefiles/ ed_conferenceo2/22.pdf

Kuznetsov, S., \& Paulos, E. (2010). Rise of the expert amateur: DIY projects, communities, and cultures. In NordiCHI' 10 Proceedings of the 6th Nordic Conference on Human-Computer Interaction: Extending Boundaries (pp. 295-304). New York, NY: ACM.

Lefteri, C. (2007). Materials: the big attraction and why material innovation is important. Ingredients, 2, 4-6.

Linnaeus, C. (1758). Systema naturae per regna tria naturae: secundum classes, ordines, genera, species, cum characteribus, diferentiis, synonymis, locis (in Latin) (10 th ed.). Stockholm: Laurentius Salvius.

Ludden, G. D. S., Schiffestein, H. N. J., \& Hekkert, P. (2012). Beyond Surprise: a longitudinal study of responses to visual - tactual incongruities in products. International Journal of Design, 1(6), 1-10.

Ludden, G. D. S., Schiffestein, H. N. J., \& Hekkert, P. (2008). Surprise as a Design Strategy.

Design Issues, 24(2), 28-38.

Nimkulrat, N. (2012). Hands-on Intellect: Integrating craft practice into design research. International Journal of Design, 6(3), 1-14.

Norman, D. (2004). Emotional Design: Why We Love (or Hate) Everyday Things. New York: Basic Book.

Ortiz, J. C. (2017). Pleasant Experiences and Sustainable Design. In Chapman, J. (Ed.). Routledge Handbook of Sustainable Product Design (pp. 367-387). Abingdon: Routledge.

Parisi, S., Rognoli, V., \& Sonneveld, M. (2017). Material Tinkering. An inspirational approach for experiential learning and envisioning in product design education, The Design Journal, 20, Sup1, S1167-S1184.

Parisi S., Spallazzo D., Ferraro V., Ferrara M., Ceconello M., Ayala-Garcia C., \& Rognoli V. (2018). Mapping ICS Materials: Interactive, Connected, and Smart Materials. In Karwowski, Waldemar, Ahram, Tareq (Eds.). Intelligent Human Systems Integration (IHSI 2018): Integrating People and Intelligent Systems. January 7-9, 2018, Dubai, United Arab Emirates, vol. 722 of the Advances in Intelligent Systems and Computing series. 
Pedgley, O., Rognoli, V., \& Karana E. (2016). Materials Experience as a Foundation for Materials and Design Education. International Journal of Technology and Design Education, 25(4), 613-630.

Pedgley, O. (2009). Influence of Stakeholders on Industrial Design Materials and Manufacturing Selection. In International Journal of Design, 3(1), 1-15.

Rognoli, V., \& Ayala-Garcia, C. (2018). Materials Activism. New hybrid scenarios between design and technology. Cuaderno 70, Cuadernos del Centro de Estudios en Diseño y Comunicación [Ensayos], 70, 105-115.

Rognoli V., Arquilla V., \& Ferrara M. (2017). ICS_Materials: materiali interattivi, connessi e smart. Material Design Journal, 2. Università di Ferrara.

Rognoli, V., Ayala-García, C., \& Parisi, S. (2016). The emotional value of Do-it-yourself materials. In Proceedings of 1oth International Conference on Design \& Emotion (pp. 233-241). Amsterdam: The Netherlands (September 2016).

Rognoli V., Bianchini M., Maffei S., \& Karana E. (2015). DIY Materials. Special Issue on Emerging Materials Experience. In Virtual Special Issue on Emerging Materials Experience, Materials and Design, vol. 86, 692-702.

Rognoli V. (2015). Dynamism and imperfection as emerging materials experiences. A case study. In Proceedings of DesForm 2015 - Aesthetics of Interaction: Dynamic, Multisensory, Wise. gth International Conference on Design and Semantics of Form and Movement, Politecnico di Milano, IT; 10/2015.

Rognoli, V., Karana, E. (2014). Toward a New Materials Aesthetic Based on Imperfection and Graceful Aging. In Karana, E., Pedgley, O., \& Rognoli, V. (Eds.) (2014). Materials Experience: Fundamentals of Materials and Design (pp. 145-154). Elsevier, UK: Butterworth-Heinemann.

Rognoli, V. Levi, M. (2011). Il senso dei materiali per il design. Milano: Franco Angeli.
Rognoli, V., (2010). A broad Survey on expressive - sensorial Characterization of Materials for Design Education. METU Journal of The Faculty of Architecture, 27(2), 287-300.

Rognoli, V.,\& Levi M. (2004). Emotions in Design through Materials An expressive - sensorial Atlas as a Project Tool for Design of Materials. In A. Kurtgözü (Ed.), Proceedings of 4 th International Conference on Design of Emotion (pp. 1-11). July 12-14. Ankara, Turkey.

Salvia, G. (2016). The satisfactory and (possibly) sustainable practice of do-it-yourself: The catalyst role of design. Journal of Design Research, 14(1), 22-41.

Sennett, R. (2008). The Craftsman. New Haven: Yale University Press.

Schifferstein, H.N.J., Hekkert, P. (Eds.) (2008). Product Experience. London: Elsevier Science.

Schön, D. (1983). The reflective practitioner: How professionals think in action. New York, NY: Basic Books.

Tanenbaum, G., Williams, A. M., Desjardins, A., \& Tanenbaum, K. (2013). Democratizing technology: pleasure, utility and expressiveness in DIY and maker practice. In Proceedings of CHI 2013. April 27 - May 2, 2013, Paris, France.

Ürgen, C. (2006). The Use and Importance of Emotional Design in Contemporary Design Practice. Master thesis, Graduate School of Engineering and Sciences of Izmir Institute of Technology.

Van Kesteren, I. E. H. (2008). Selecting materials in product design (Doctoral dissertation). Delft University of Technology, Delft, The Netherlands.

Van Kesteren, I. E. H., Stappers, P. J., Kandachar, P. V. (2005). Representing Product Personality in Relation to Materials in the Design Problem. In Proceedings of the International Conference of the Nordic Design Research Society, 29-31 May, Kopenhagen, Denmark. 